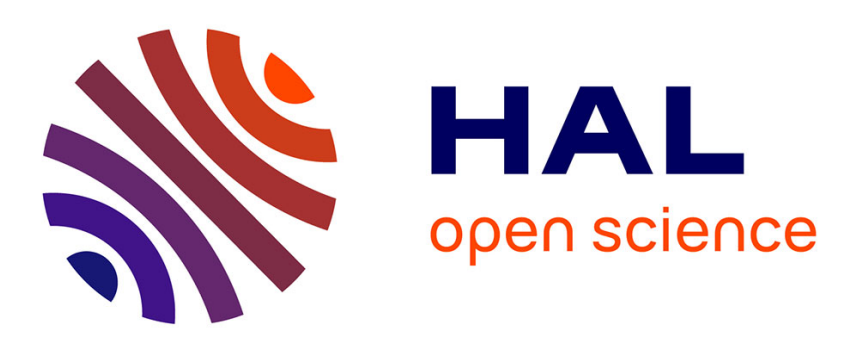

\title{
Towards the control of tensegrity mechanisms for variable stiffness applications: a case study
}

Quentin Boehler, Salih Abdelaziz, Marc Vedrines, Philippe Poignet, Pierre Renaud

\section{- To cite this version:}

Quentin Boehler, Salih Abdelaziz, Marc Vedrines, Philippe Poignet, Pierre Renaud. Towards the control of tensegrity mechanisms for variable stiffness applications: a case study. EUCOMES: European Conference on Mechanism Science, Sep 2016, Nantes, France. pp.163-171, 10.1007/978-3-319-441566_17. lirmm-01643092

\section{HAL Id: lirmm-01643092 https://hal-lirmm.ccsd.cnrs.fr/lirmm-01643092}

Submitted on 17 Dec 2018

HAL is a multi-disciplinary open access archive for the deposit and dissemination of scientific research documents, whether they are published or not. The documents may come from teaching and research institutions in France or abroad, or from public or private research centers.
L'archive ouverte pluridisciplinaire HAL, est destinée au dépôt et à la diffusion de documents scientifiques de niveau recherche, publiés ou non, émanant des établissements d'enseignement et de recherche français ou étrangers, des laboratoires publics ou privés. 


\title{
Toward the control of tensegrity mechanisms for variable stiffness applications: a case study
}

\author{
Q. Boehler ${ }^{1}$, S. Abdelaziz ${ }^{2}$, M. Vedrines ${ }^{1}$, P. Poignet ${ }^{2}$ and P. Renaud ${ }^{1}$ \\ ${ }^{1}$ ICube, Strasbourg, France, e-mail: q.boehlereunistra. fr \\ ${ }^{2}$ LIRMM, Montpellier, France
}

\begin{abstract}
Cable-driven tensegrity mechanisms can be considered to control at the same time position and stiffness. Adequate control solutions have however not been proposed yet. This paper thus focuses on the development of an original control strategy using a tension distribution algorithm adapted from related work on cable-driven mechanisms. The algorithm is being used to modify the mechanism configuration together with its stiffness through the level of prestress in the system, which constitutes a step toward the exploitation of such mechanisms for variable stiffness applications. Simulations show encouraging results on the stiffness variation capacity of the presented mechanism.
\end{abstract}

Key words: Tensegrity mechanism, cable-driven mechanism, tensegrity modeling and control, tension distribution algorithm, variable stiffness.

\section{Introduction}

A tensegrity mechanim is a self-stressed system comprising a set of compressed bars in a set of tensioned elements [10] that can be either cables or springs. Actuation of the mechanism allows for device reconfiguration and such kind of mechanism has been demonstrated to be of interest in applications ranging from mobile robotics [9] to manipulators [1]. Besides, so-called tensegrity systems have been considered for variable stiffness applications [3], as the stiffness along specific directions can be dramatically modified through the level of prestress within its components. In this case, elastic cables or springs are preferred in order to bring compliance to the system around a fixed configuration. Introducing tensegrity mechanisms in which both the configuration and the stiffness can be controlled seems of great interest for robotic applications where variable stiffness is desired. This paper is thus focused on the control of such tensegrity mechanisms, an aspect which has not been yet investigated in the literature.

The modeling of tensegrity mechanisms is complex, as the tensions in the cables or springs have to remain positive, and static conditions must be taken into account as shown in [4]. Cable-actuated tensegrity mechanisms therefore interestingly share 


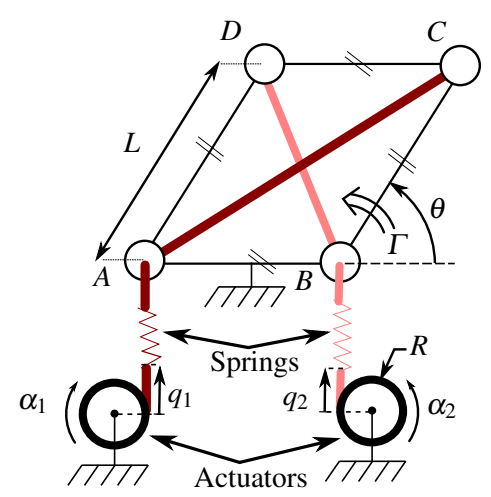

Fig. 1 Cable-driven planar tensegrity mechanism.

properties with cable-driven robots. A cable-driven robot is composed of a platform connected to the base by cables which lengths are modified in a coordinated way to control the platform pose. As a cable can only be tensioned, unidirectional constraints exist, contrary to classical parallel manipulators in which the kinematic redundancy can be managed through bidirectional constraints [6]. In this paper, we propose to take advantage of recent developments in the control of cable-driven robots to develop a control strategy of tensegrity mechanism for variable stiffness applications.

The analysis we perform is divided into two steps. First, the position control of cable-actuated tensegrity mechanisms is investigated. An original controller is proposed based on so-called tension distribution algorithms [7]. Second, a stiffness modulation strategy through cable prestress is proposed. This strategy is implemented on an original planar cable-driven tensegrity mechanism. Thanks to the architecture of the mechanism, meaningful simulations results related to the configuration and stiffness control are obtained.

In section 2 , the considered system is presented together with its modeling. In Section 3, a description of the control strategy is provided before introducing control behavior through simulation in section 4 . Conclusions and future works are developed in section 5 .

\section{System description and modeling}

\subsection{Description}

The considered system is depicted in Fig. 1. It is composed of one static and three moving bars of length $L$, articulated in four pin joints $A, B, C$ and $D$. The resulting parallelogram linkage is actuated by two cables attached in $C$ and $D$ and passing through $A$ and $B$. The system is indeed a tensegrity mechanism as the three bars 
are compressed by two tensioned actuated cables. As a parallelogram linkage, this system exhibits one degree of freedom. The mechanism configuration is represented by the angle $\theta$.

Two linear springs of stiffness $k$ are integrated along each cable to bring compliance to the system. The resulting angular stiffness of the end-effector $B C$ is denoted $K_{\theta}$. The distances $A C$ and $B D$ are respectively denoted $l_{1}$ and $l_{2}$ and can be computed as

$$
\left\{\begin{array}{l}
l_{1}=2 L \cos (\theta / 2) \\
l_{2}=2 L \sin (\theta / 2)
\end{array}\right.
$$

The lengths of the cables, denoted $q_{1}$ and $q_{2}$ (see Fig. 1), are controlled by two actuators of angular positions $\alpha_{1}$ and $\alpha_{2}$ through pulleys of radius $R$. In the following $\mathbf{q}=\left[q_{1}, q_{2}\right]^{T}, \boldsymbol{\alpha}=\left[\alpha_{1}, \alpha_{2}\right]^{T}$ and therefore $\mathbf{q}=-R \boldsymbol{\alpha}$.

\subsection{Kinematic and static modeling}

As a tensegrity composed of deformable springs, this system is submitted to selfequilibrium constraints that have to be considered together with the kinematic ones [4]. Using the same energetic approach than in [2], the equilibrium equation is expressed as

$$
\frac{\partial U}{\partial \theta}=0
$$

with $U$ the potential energy assumed to be fully stored in the springs. The angular stiffness $K_{\theta}$ is computed using the second derivative of $U$ with respect to $\theta$ so that

$$
K_{\theta}=\frac{\partial^{2} U}{\partial \theta^{2}}
$$

The two cables are used to control the configuration of the parallelogram, so we consider $\mathbf{q}$ as the articular variables. The system is kinematically redundant, i.e. each configuration can be reached with different levels of prestress. It also means that Eq. (2) can be satisfied for different values of $K_{\theta}$. Two variables are required to fully represent the system state, namely its configuration $\theta$ and its angular stiffness $K_{\theta}$. In the following, we thus designate the vector $\mathbf{X}=\left[\theta, K_{\theta}\right]^{T}$ as the operational variables, as they are the variables to be simultaneously modified for a given task. Eq. (2) and (3) lead to the so-called Forward Static Model, i.e. the expression of $\mathbf{X}$ with respect to $\mathbf{q}$ :

$$
\left\{\begin{array}{l}
\theta=2 \arctan \left(q_{2} / q_{1}\right) \\
K_{\theta}=\frac{k L}{2} \frac{q_{1}^{2}+q_{2}^{2}}{\sqrt{q_{1}^{2}+q_{2}^{2}}}
\end{array}\right.
$$




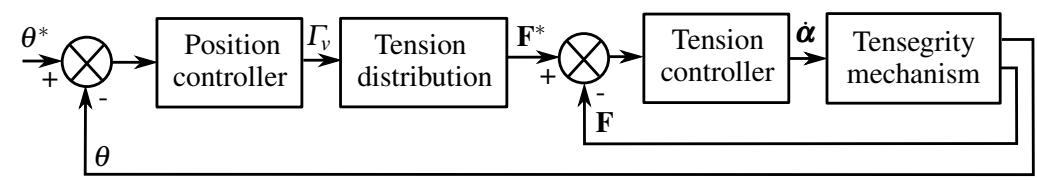

Fig. 2 Control strategy.

The first equation in (4) can be retrieved using the virtual work principle that gives a relationship between the cable tension set $\mathbf{F}=\left[F_{1}, F_{2}\right]^{T}$ and the torque $\Gamma$ applied on the output bar $B C$ (see Fig. 1):

$$
L \sin (\theta / 2) F_{1}-L \cos (\theta / 2) F_{2}=\Gamma
$$

that can be noted

$$
\mathbf{W F}=\Gamma
$$

with $\mathbf{W}=[L \sin (\theta / 2),-L \cos (\theta / 2)]$. The equilibrium is reached when $\Gamma=0$, and then the first equation in (4) can be retrieved from (6) knowing that $F_{i}=k\left(l_{i}-q_{i}\right)$ for the $i$-th spring. The reader should notice that two forces are used to generate a single torque. This redundancy, specific to cable-driven systems, means that a given torque can be generated for several couple of forces i.e. for several levels of prestress within the system. This specificity in the mechanism modeling is the starting point of the strategy we propose in order to reconfigure the system while controlling its prestress and thus its stiffness.

\section{Control strategy}

\subsection{Tension distribution algorithm}

The system position has to be controlled while ensuring that the tension set $\mathbf{F}$ remains in the user-defined positive interval bounded by $\mathbf{F}_{\min }$ and $\mathbf{F}_{\mathbf{m a x}}$. In this paper, we propose the control strategy depicted in Fig. 2 adapted from [8]. An external loop controls the reference configuration $\theta^{*}$ using a position controller that generates a virtual torque $\Gamma_{v}$ to be applied to the system. This torque is used as an input to the tension distribution block that solves

$$
\mathbf{W F}^{*}=\Gamma_{v} \text {, subjected to } \mathbf{F}_{\min } \leq \mathbf{F}^{*} \leq \mathbf{F}_{\max }
$$

The inner loop then uses a tension controller to feed the actuators with the velocities $\dot{\boldsymbol{\alpha}}$. It can be proved from [7] that the solution to Eq. (7) is of the form

$$
\mathbf{F}^{*}=\mathbf{W}^{+} \Gamma_{v}+\mathbf{N}_{\mathbf{W}} \lambda
$$


where $\mathbf{W}^{+}$denotes the Moore-Penrose pseudoinverse of $\mathbf{W}, \mathbf{N}_{\mathbf{W}}$ a basis of $\mathbf{W}$ nullspace, and $\lambda$ a scalar to be chosen so that the inequality in Eq. (7) is satisfied, namely that

$$
\mathbf{F}_{\min }-\mathbf{W}^{+} \Gamma_{v} \leq \mathbf{N}_{\mathbf{W}} \lambda \leq \mathbf{F}_{\max }-\mathbf{W}^{+} \Gamma_{v}
$$

which is equivalent to

$$
\lambda_{\min } \leq \lambda \leq \lambda_{\max }
$$

The existence of a solution to Eq. (7) depends on the value of the torque $\Gamma_{v}$ to be applied. The feasibility of $\Gamma_{v}$ is verified and its value is saturated accordingly using the approach of [5] to ensure the existence of a solution set. Then using the approach described in [7], the set $\Lambda=\left\{\lambda \mid \lambda_{\min } \leq \lambda \leq \lambda_{\max }\right\}$ can be computed.

The second term of (8) corresponds to the level of prestress within the system. This latter does not modify the current equilibrium configuration of the system as it is chosen within $\mathbf{W}$ nullspace, so that it generates no torque $\Gamma$ on the end-effector. The modulation of $\lambda$ within $\Lambda$ has thus a direct impact on the stiffness of the system and no influence on the system configuration. This property is at the center of the stiffness modulation strategy.

\subsection{Stiffness modulation through prestress}

In existing applications such as described in [7], the tension distribution algorithm is only used to provide a single feasible solution to Eq. (7) among the infinite number of solutions in $\Lambda$. The scalar $\lambda$ can be chosen as the center of gravity of $\Lambda$, which has been proved to be continuous along continuous trajectories [7]. In that case, it means that $\lambda=\left(\lambda_{\max }+\lambda_{\min }\right) / 2$, so that the level of prestress is chosen as far away as possible from the upper and lower bounds of $\mathbf{F}^{*}$.

In this paper, our idea is to modulate the choice of $\lambda$ in $\Lambda$ to modify the level of prestress and thus the stiffness. Around an equilibrium configuration (i.e. $\Gamma_{v}=0$ ), the first term of Eq. (8) vanishes and it becomes

$$
\mathbf{F}^{*}=\mathbf{N}_{\mathbf{W}} \lambda
$$

where $\mathbf{N}_{\mathbf{W}}=[-L \cos (\theta / 2),-L \sin (\theta / 2)]^{T}$ is a basis of $\mathbf{W}$ nullspace. As $F_{i}=k\left(l_{i}-\right.$ $q_{i}$ ) for the $i$-th spring, Eq. (11) can be combined with Eq. (4) and (1) to give

$$
K_{\theta}=\frac{L^{2}}{2}(\lambda+2 k)
$$

The stiffness $K_{\theta}$ can thus be explicitly controlled through $\lambda$ using the tension distribution algorithm. This constitutes an original use of the algorithm in order to modify the stiffness of the mechanism at a fixed configuration through a prestress modulation. 


\section{Simulations}

In the following, the behavior obtained with the proposed approach is analyzed through a trajectory tracking simulation. In addition, the stiffness modulation is assessed to provide a first insight of the system ability to be controlled in position and stiffness simultaneously. We choose $L=70 \mathrm{~mm}, k=0.27 \mathrm{~N} / \mathrm{mm}$ and $R=8.4 \mathrm{~mm}$ as consistent values and to ensure future experimental validations. Thanks to the particular arrangement of the elements within a tensegrity mechanism, important loads can be supported using only slender and lightweight components. The weights and inertia within the system are therefore neglected. The control scheme of Fig. 2 is implemented in Matlab Simulink (The Mathworks, Inc.) in which the tensegrity mechanism is represented by its static model. The dynamics of the system as well as those of the actuators are neglected. These assumptions are justified by the trajectories to be tracked in the simulation, which exhibits low accelerations.

\subsection{Trajectory tracking}

The simulated trajectory aims at demonstrating the reconfiguration capacity of the system, as well as the impact of the configuration on $\Lambda$. For didactic purposes, we also show the impact of the tensions bounds on the system behavior. The trajectory is chosen accordingly as commented in the following.

The simulation results are illustrated in Fig. 3. During the first $20 \mathrm{~s}$, the mechanism is driven from $\theta^{*}=90^{\circ}$ to $\theta^{*}=45^{\circ}$ with constant bounds on $\mathbf{F}^{*}$ of $4 \mathrm{~N}$ and $10 \mathrm{~N}$. For the next $5 \mathrm{~s}$, the upper bound is linearly increased to $20 \mathrm{~N}$ for $\theta^{*}=45^{\circ}$. During the last $5 \mathrm{~s}$, both bounds on $\mathbf{F}^{*}$ and $\theta^{*}$ are fixed, so that neither the configuration nor the tensions are modified by the algorithm.

The cable tensions are equal at $\theta^{*}=90^{\circ}$ thanks to the symmetrical arrangement of the cables, and they are equal to $\left(F_{\max }+F_{\min }\right) / 2$, the center of gravity of the feasible tensions. Between 0 and $20 \mathrm{~s}, \Lambda$ shrinks as the cables tensions get closer to their bounds. The limit of the tension distribution for these bounds is met at $45^{\circ}$ where $F_{1}=F_{\max }$ and $F_{2}=F_{\min }$, and $\Lambda$ is then reduced to a single value. Finally, when $F_{\max }$ is increased from 10 to $20 \mathrm{~N}$, the tensions continuously adapt to the new bounds thus keeping the same configuration for different tension sets.

These results show the ability of the system to track a trajectory in $\theta$, but also to reach the same configuration for different tension sets.

\subsection{Stiffness modulation}

In order to demonstrate the stiffness modulation ability of the system, the angle is set to a constant value: $\theta^{*}=90^{\circ}$ for the first test and then $\theta^{*}=75^{\circ}$. The scalar $\lambda$ 
is imposed so that $K_{\theta}$ lineary goes from 0.5 to $1 \mathrm{Nm} / \mathrm{rad}$ during $5 \mathrm{~s}$ (see Eq. (12)). The results are depicted in Fig. 4.

As already noticed in the previous simulation, the cable tensions are equal all along the modulation for $\theta=90^{\circ}$. This is no more the case for $\theta=75^{\circ}$. In both cases, $\lambda$ is kept in $\Lambda$ so that the cable tensions remain between the preset bounds (here between 4 and $20 \mathrm{~N}$ ). It should be noticed that for this particular mechanism, the results may be counter-intuitive as the stiffness is increased when the prestress decreases in the cables. These simulation results show the original use of the tension distribution algorithm coupled with Eq. (12) to provide a stiffness modulation through a variation of the prestress within the mechanism.

(a)
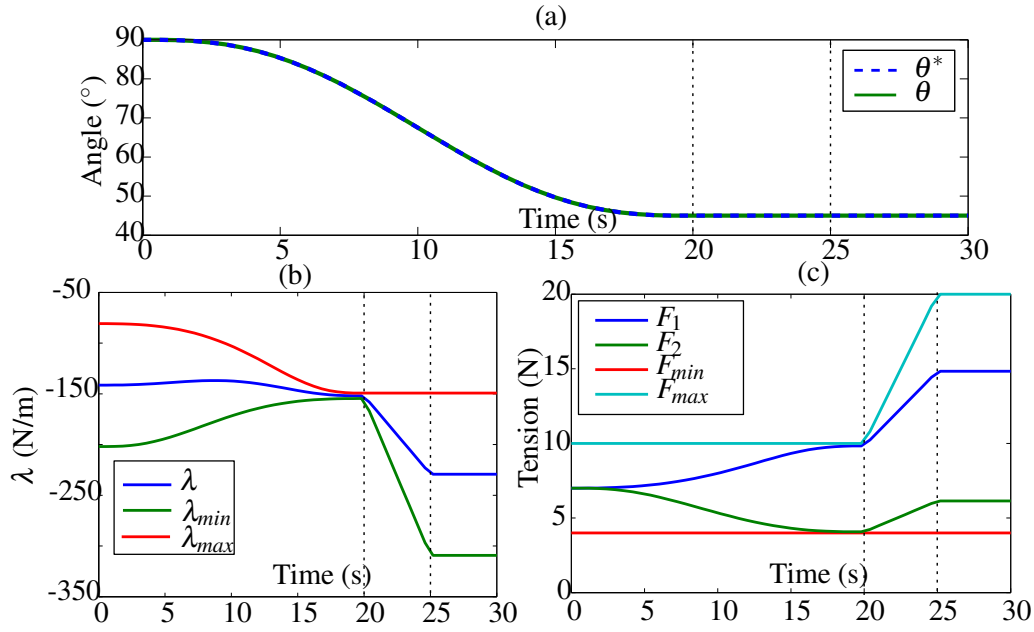

Fig. 3 Simulation on a trajectory tracking between $\theta^{*}=90^{\circ}$ to $\theta^{*}=45^{\circ}$. (a) Reference and current angle ; (b) Evolution of $\lambda$ and $\Lambda$; (c) Evolution of the cable tensions and their bounds.
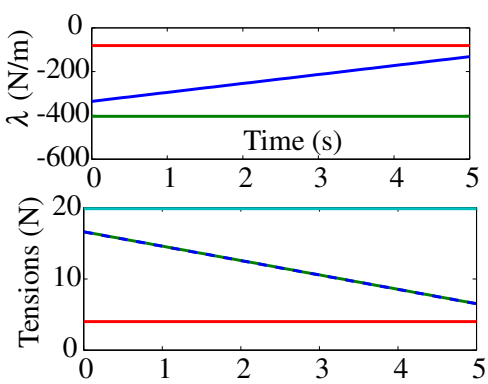

(a)
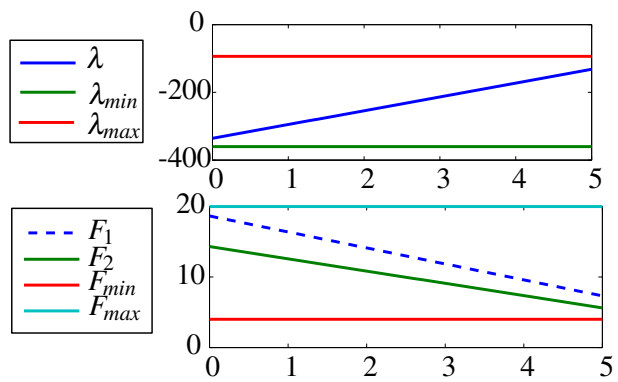

(b)

Fig. 4 Simulation of a stiffness linear variation between $K_{\theta}=0.5$ and $1 \mathrm{Nm} / \mathrm{rad}$ during $5 \mathrm{~s}$ for (a) $\theta=90^{\circ}$ and (b) $\theta=75^{\circ}$. Top graphs represent the evolution of $\lambda$ and $\Lambda$, bottom ones the evolution of the cable tensions and their bounds. 


\section{Conclusions and perspectives}

In this paper, a novel control strategy for cable-actuated tensegrity mechanisms is proposed. Existing works on cable-driven robots are exploited to design an adapted control strategy. This strategy is implemented on a 1-DOF tensegrity mechanism which state is defined as its configuration together with its stiffness about its DOF. Using an original adaptation of the tension distribution algorithm, both the configuration and the stiffness can be modified. Simulations notably show encouraging results on the stiffness variation capacity of the system. These results clearly emphasize the potential of this mechanism and the control strategy for variable stiffness robotic devices.

Further works will be carried out on the experimental validation of these results as well as on the development of alternate control strategies for further applications.

Acknowledgements This work was supported by French state funds managed by the ANR within the Investissements d'Avenir programme (Robotex ANR-10-EQPX-44, Labex CAMI - ANR-11LABX-0004) and by the Région Alsace and Aviesan France Life Imaging infrastructure.

\section{References}

1. Aldrich, J., Skelton, R., Kreutz-Delgado, K.: Control synthesis for a class of light and agile robotic tensegrity structures. In: Proceedings of the 2003 IEEE ACC, vol. 6, pp. 5245-5251 vol.6 (2003)

2. Arsenault, M., Gosselin, C.M.: Kinematic, static, and dynamic analysis of a planar one-degreeof-freedom tensegrity mechanism. ASME J Mech Design 127(6), 1152-1160 (2005)

3. Azadi, M., Behzadipour, S., Faulkner, G.: A variable spring using a tensegrity prism. In: Proceedings of the 2009 ASME IDETC/CIE, pp. 35-43 (2009)

4. Boehler, Q., Charpentier, I., Vedrines, M.S., Renaud, P.: Definition and computation of tensegrity mechanism workspace. ASME J Mech Robot 7(4), 044,502 (2015)

5. Bouchard, S., Gosselin, C., Moore, B.: On the ability of a cable-driven robot to generate a prescribed set of wrenches. ASME J Mech Robot 2(1), 011,010 (2009)

6. Ebert-Uphoff, I., Voglewede, P., et al.: On the connections between cable-driven robots, parallel manipulators and grasping. In: Proceedings of the 2004 IEEE ICRA, vol. 5, pp. 4521-4526 (2004)

7. Gouttefarde, M., Lamaury, J., Reichert, C., Bruckmann, T.: A versatile tension distribution algorithm for $n-D O F$ parallel robots driven by $n+2$ cables. IEEE Trans. Robot. 31(6), 14441457 (2015)

8. Khosravi, M.A., Taghirad, H.D.: Robust PID control of fully-constrained cable driven parallel robots. Mechatronics 24(2), 87 - 97 (2014)

9. Koizumi, Y., Shibata, M., Hirai, S.: Rolling tensegrity driven by pneumatic soft actuators. In: Proceedings of the 2012 IEEE ICRA, pp. 1988-1993 (2012)

10. Motro, R.: Tensegrity: Structural Systems for the Future. Elsevier (2003) 